\title{
Development and evaluation of internal amplification controls for use in a real-time duplex PCR assay for detection of Campylobacter coli and Campylobacter jejuni
}

Correspondence

Luke Randall

l.randall@vla.defra.gsi.gov.uk

Received 16 July 2009

Accepted 8 October 2009

\author{
Luke Randall, Fabrizio Lemma, John Rodgers, Ana Vidal \\ and Felicity Clifton-Hadley \\ Veterinary Laboratories Agency (Weybridge), New Haw, Addlestone, Surrey KT15 3NB, UK
}

\begin{abstract}
A common problem of both conventional and real-time PCR assays is failure of DNA amplification due to the presence of inhibitory substances in samples. In view of this, our aim was to develop and evaluate internal amplification controls (IACs) for use with an existing duplex real-time PCR assay for Campylobacter coli and Campylobacter jejuni. Both competitive and non-competitive IACs were developed and evaluated. The competitive approach involved a DNA fragment of the coding region of the fish viral haemorrhagic septicaemia virus, flanked by the mapA PCR primers, whilst the non-competitive approach utilized an extra set of universal 16S rDNA primers. Both IAC-PCR assay types were evaluated using cultures of Campylobacter and chicken caecal content samples. Both IACs were sensitive to caecal inhibitors, making them suitable for detecting inhibition which could lead to false-negatives. Results showed that both IACs at optimum concentrations worked well without reducing the overall sensitivity of the PCR assay. Compared to culture, the optimized competitive IAC-PCR assay detected 45/47 positives (sensitivity $93.6 \%$, specificity $80.1 \%$ ); however, it had the advantage over culture in that it could detect mixed infections of C. coli and C. jejuni and was capable of giving a result for a sample within a day.
\end{abstract}

\section{INTRODUCTION}

Campylobacter enteritis is the most common cause of acute bacterial diarrhoea worldwide (Linton et al., 1997; Keramas et al., 2004) and poultry and poultry products are considered important sources of human campylobacteriosis (Genigeorgis et al., 1986; Harris et al., 1986; Deming et al., 1987; Evans, 1992; Keramas et al., 2004).

Isolation and identification of Campylobacter by conventional culture methods is slow (Keramas et al., 2004) and there is a need for more rapid techniques. There are existing PCR assay protocols for detection of Campylobacter in water (Kirk \& Rowe, 1994), in some dairy products (Wegmuller et al., 1993), in meat (Giesendorf et al., 1992) and in poultry (Lund et al., 2003), and in human faeces (Linton et al., 1997; Lawson et al., 1999), as well as many papers that describe PCR assay identification of Campylobacter from pure culture (Oyofo et al., 1992; Comi et al., 1996; Best et al., 2003). Similarly, there are multiplex PCR assays described for the detection of Campylobacter coli and Campylobacter jejuni (Denis et al.,

Abbreviations: FV-IAC, fish haemorrhagic virus internal amplification control; IAC, internal amplification control.
1999; Best et al., 2003) and for the detection of C. coli, Campylobacter fetus subsp. fetus, C. jejuni, Campylobacter lari and Campylobacter upsaliensis (Wang et al., 2002).

One of the limitations to the widespread use of PCR is that PCR assays are usually run without internal amplification controls (IACs). Whilst external positive and negative controls may give some assurance that the PCR assay is effective, they do not give assurance of an individual negative result. A negative result may be a true-negative result, or a false-negative due to the presence of inhibitory substances within the sample (Hoorfar et al., 2004). Faecal or caecal samples in particular can contain substances that are inhibitory to PCR assays (Lund \& Madsen, 2006). For a PCR assay to be validated through a multicentre collaborative trial, it must contain an IAC (Lund \& Madsen, 2006), although the inclusion of IACs in all PCR assays would give assurance of reliable results.

There have been many studies that have looked into the development and use of IACs in PCR assays, as recently reviewed by Hoorfar et al. (2004). One such study focused in particular on an IAC for conventional and real-time PCR assay for detection of Campylobacter species in 
chicken faecal samples (Lund \& Madsen, 2006). However, we were not aware of any study that evaluated different types of IACs in a real-time duplex PCR assay for C. coli and C. jejuni.

In view of the need to identify the two most important Campylobacter species (C. coli and C. jejuni) rapidly from caecal or faecal samples, in this study we used an existing real-time PCR assay for identifying C. coli and C. jejuni (Best et al., 2003), but modified it by incorporation of one of two different IACs. We evaluated an existing in-house non-competitive $16 \mathrm{~S}$ rDNA IAC which incorporated a set of universal $16 \mathrm{~S}$ rDNA primers and also designed and evaluated a competitive IAC based on a haemorrhagic fish virus sequence flanked by one of the existing primer sets (Best et al., 2003). Both IACs were evaluated with cultures, with spiked caecal samples, with field caecal samples and with PCR assay inhibitor derived from caecal contents.

\section{METHODS}

Bacterial strains. Campylobacter strains were grown as previously described (Best et al., 2003). The following cultures obtained from the National Collection of Type Cultures (NCTC) were used as positive and negative control strains for PCR optimization: C. coli NCTC 12143; C. jejuni NCTC $11351^{\mathrm{T}}$; C. lari NCTC $11352^{\mathrm{T}}$; C. fetus subsp. fetus NCTC $10842^{\mathrm{T}}$; Campylobacter hyointestinalis NCTC $11608^{\mathrm{T}}$; Campylobacter sputorum subsp. bubulus NCTC $11367^{\mathrm{T}}$; and Arcobacter cryaerophilus NCTC $11885^{\mathrm{T}}$. Crude cell lysates were used to provide the DNA from pure cultures for PCR assay. In brief, crude DNA lysates were prepared by taking growth (one $10 \mu \mathrm{l}$ loopful) from a non-selective agar plate into $700 \mu \mathrm{l}$ sterile distilled water in a sterile Eppendorf tube and vortexing to give a suspension. This suspension was placed in a boiling water bath for $10 \mathrm{~min}$, centrifuged for $3 \mathrm{~min}$ at $16200 \mathrm{~g}$ and the supernatant was stored at $4{ }^{\circ} \mathrm{C}$ (for up to 1 month) prior to use.

PCR primers and conditions, probe and IAC sequences. The primer and probe sequences for the $c e u E$ gene (which encodes a lipoprotein) of C. coli and the mapA gene (which encodes an outermembrane protein) of $C$. jejuni have been described previously (Best et al., 2003). The duplex real-time PCR assay conditions were as described by Best et al. (2003) with the following exceptions. Each PCR assay reaction was $25 \mu \mathrm{l}$ and comprised $12.5 \mu \mathrm{l}$ Qiagen HotStarTaq PCR assay Master Mix (cat. no. 203445), $3.5 \mu \mathrm{l} 25 \mathrm{mM}$ $\mathrm{MgCl}_{2}$ solution, $0.5 \mu \mathrm{l}$ each primer at $20 \mu \mathrm{M}, 0.5 \mu \mathrm{l}$ each probe at $5 \mu \mathrm{M}$, and nuclease-free water to take the volume to $23 \mu \mathrm{l}$. Template was $2 \mu \mathrm{l}$ to make the final volume to $25 \mu \mathrm{l}$.

The non-competitive $16 \mathrm{~S}$ rDNA IAC-PCR assay included an extra set of universal $16 \mathrm{~S}$ rDNA primers with the sequences $16 \mathrm{~S}-\mathrm{F} 5^{\prime}$ ACTACGTGCCAGCAGCC-3' and 16S-R 5'-GGACTACCAGGGTATCTAATCC- $3^{\prime}$, and these were added to the PCR assay Master Mix at the same concentration as the other primers. The non-competitive 16S rDNA IAC-PCR assay Master Mix also included a ROX probe specific for the $16 \mathrm{~S}$ rDNA amplicon and the sequence of this probe was $5^{\prime}$-ROX-TGTTTGCTCCCCACGCTTTCGCA-BHQ2-3'. For the $16 S$ rDNA IAC-PCR assay, the amount of water in each PCR assay reaction was reduced to allow for addition of extra primers and probe.

The competitive fish haemorrhagic virus IAC (FV-IAC) comprised a $60 \mathrm{bp}$ sequence from the fish haemorrhagic virus sequence (accession no. X66134) flanked by the mapA gene PCR primers. The complete sequence was as follows, with the fish virus sequence in bold underline: 5 '-CTGGTGGTTTTGAAGCAAAGATTAGGTCAACTCAGGTGTCCTCATGAATTTGAAGACATAAACAAGGGACTGGTCTTATTTATATAAACGCACTTTAGACACTGGTATTG-3'. The FVIAC PCR assay Master Mix also included a ROX probe specific for the fish virus sequence amplicon and the sequence of this probe was 5'-ROX-TGA $[+$ G]GA[+ C $]$ AC $[+$ C $]$ TGAG $[+$ T $]$ TGACCT-BHQ2-3', where + denotes locked nucleic acids. For the FV-IAC PCR assay, the amount of water in each PCR assay reaction was reduced to allow for addition of the IAC and the probe.

All primers and probes and the FV-IAC were obtained from SigmaAldrich. All samples were analysed using a Stratagene MX3000P machine. Cycling conditions were one cycle of $95{ }^{\circ} \mathrm{C}$ for $15 \mathrm{~min}$, followed by 40 cycles of $94{ }^{\circ} \mathrm{C}$ for $30 \mathrm{~s}, 60{ }^{\circ} \mathrm{C}$ for $30 \mathrm{~s}$ and $72{ }^{\circ} \mathrm{C}$ for $30 \mathrm{~s}$. Fluorescence was read at the $60{ }^{\circ} \mathrm{C}$ position.

Caecal samples and PCR assay inhibitor. Chicken caeca were collected from broiler flocks at time of slaughter as part of a 3 year national prevalence survey of Campylobacter in broilers in the UK. A fresh pooled caecal sample for each flock was produced by suspending $0.02 \mathrm{~g}$ caecal contents from each of 10 individual birds in $2 \mathrm{ml} 0.1 \mathrm{M}$ PBS ( $\mathrm{pH}$ 7.2). The campylobacter status of these flocks had been determined by the culture methods specified by the EU (ISO, 2006).

To determine the limit of detection of the PCR assay with and without an IAC, a total of 16 spiked caecal samples were prepared from fresh pooled caecal contents from two flocks. Eighteen further naturally infected caecal samples were also used to compare the PCR assay with and without different IACs. Finally, the chosen PCR-IAC method was assessed on a further 52 pooled caecal samples and the results were compared to those of culture.

DNA from both naturally infected and spiked caecal samples was extracted using an ExtractMaster Fecal DNA Extraction kit (Epicentre Biotechnologies), with $0.25 \mathrm{ml}$ of the caecal contents suspended in $0.75 \mathrm{ml}$ PBS and then DNA extracted according to the manufacturer's instructions. Extraction controls comprising caecal samples known to be positive for Campylobacter were run with each batch of DNA extracts from caecal contents.

DNA from both crude culture lysates and caecal extractions was quantified for a representative number of samples using a NanoDrop 1000 (Thermo Fisher Scientific).

Caecal PCR assay inhibitor solution was made by suspending caecal contents in distilled water $(50: 50, \mathrm{w} / \mathrm{v})$, mixing, centrifuging in a microcentrifuge for $3 \mathrm{~min}$ at 13000 r.p.m. and then storing the supernatant (Caecal PCR assay inhibitor solution) at $-20{ }^{\circ} \mathrm{C}$ until required for use. In experiments that included Caecal PCR assay inhibitor solution, this was added at $1 \mu \mathrm{l}$ per reaction and the volume of water in each reaction was reduced commensurately.

Spiking caecal samples. Caecal samples from flocks found to be free from Campylobacter by culture were spiked with strains $C$. jejuni NCTC $11351^{\mathrm{T}}$ and C. coli NCTC 12143. Strains were grown from frozen $\left(-80{ }^{\circ} \mathrm{C}\right)$ stocks in $10 \%$ glycerol broth (Oxoid) on $7 \%$ sheep blood agar with $0.1 \%$ actidione (Oxoid) and pure culture was harvested into PBS to McFarland standard 4 to give $\sim \log _{10} 9$ c.f.u. $\mathrm{ml}^{-1}$. C. coli and C. jejuni in PBS were then mixed $1: 1$ to give $\sim \log _{10}$ 8 c.f.u. $\mathrm{ml}^{-1}$ of each, and further diluted to give $\sim \log _{10} 6,4,2$ c.f.u. $\mathrm{ml}^{-1}$ of each Campylobacter strain. Respective dilutions $(0.1 \mathrm{ml})$ were then added to duplicate $0.9 \mathrm{~g}$ caecal content sample to give samples spiked with $\sim \log _{10} 7,5,3$ and 1 c.f.u. Campylobacter (g caecal sample $)^{-1}$. Duplicate sets of spiked caecal samples were prepared on two different occasions and the DNA was extracted from them, so in all four replicates of spiked samples and four different dilutions were tested, a total of 16 spiked samples. 
Enumeration of Campylobacter in spiked caecal samples. To enumerate the number of Campylobacter in the spiked caecal samples, the original PBS suspensions used to create the spiked caecal samples were serially diluted in PBS until a $10^{-9}$ dilution was created and then $100 \mu \mathrm{l}$ of each dilution was plated onto duplicate modified Charcoal Cefoperazone Deoxycholate agar (Oxoid) plates. Plates were incubated at $41.5 \pm 1{ }^{\circ} \mathrm{C}$ in a microaerobic atmosphere $\left(84 \% \mathrm{~N}_{2} ; 10 \% \mathrm{CO}_{2} ; 6 \%\right.$ $\mathrm{O}_{2}$ ) for $48 \mathrm{~h}$ and the numbers of typical Campylobacter colonies were counted. The most dilute pair of plates that contained a mean of between 15 and 200 typical colonies was used to calculate the number of Campylobacter c.f.u. (g caecal content $)^{-1}$. A colony from each plate used for calculation was purified and confirmed as Campylobacter sp. using the methods previously described (ISO, 2006).

Optimization of the PCR assay. IACs are known to have the capacity to reduce the sensitivity of PCR assays, particularly for competitive IACs (Hoorfar et al., 2004). In order to minimize the competitive effect of the FV-IAC, it was tested at dilutions of $10^{-5}$, $10^{-6}$ and $10^{-7}$ (from 200 down to $2 \mathrm{pmol}$ ) and the strength of the $C$. coli and C. jejuni signals was compared to the PCR assay with no IAC. In a similar way, for the non-competitive IAC, the $16 \mathrm{~S}$ rDNA primers were tested at a standard concentration, as well as at 1/2,1/4 and 1/10 standard concentrations. This initial optimization was performed using cell lysate preparations derived from the positive and negative control cultures.

Evaluation of PCR assays. Once the different PCR assays were optimized for use with pure culture, they were evaluated with C. coli and $C$. jejuni cell lysates at doubling dilutions from neat to $1 / 1024$, spiked $(n=16)$ and natural $(n=18)$ chicken caecal DNA extracts and with 'Caecal PCR assay inhibitor solution'. The PCR assay type that overall gave the most sensitive and specific results was then used to test a further 52 natural chicken caecal DNA extracts and the results were compared to those obtained by culture.

The effect of Caecal PCR assay inhibitor solution for inhibiting the IAC components as well as the C. coli and C. jejuni components of the PCR assays was determined for $1 \mu \mathrm{l}$ Caecal PCR assay inhibitor solution added to each $25 \mu \mathrm{l}$ PCR assay reaction. The Caecal PCR assay inhibitor solution was tested neat, and at doubling dilutions ranging down to $1 / 2048$.

Testing natural caecal samples for the presence of Campylobacter. For 52 natural caecal samples, detection of Campylobacter by PCR assay was compared to detection by culture. The Campylobacter status of these samples was determined by the culture methods specified by the EU baseline survey for monitoring of Campylobacter prevalence in broiler flocks (ISO, 2006).

Adjusting $\boldsymbol{C}_{\mathbf{t}}$ values to relative values. The output from the Stratagene PCR assay machine is such that the higher the $C_{\mathrm{t}}$ value (threshold cycle), the weaker the signal. For PCR assay of cultures, samples with $C_{\mathrm{t}}$ values above 32 are considered negative (Best et al., 2003). To make graphics more meaningful (e.g. so stronger signals appeared as higher values rather than lower values), $C_{\mathrm{t}}$ values were adjusted to relative $C_{\mathrm{t}}$ values so that higher values would correlate to stronger signals. This adjustment was made using the formula $\left(40 / C_{\mathrm{t}}\right.$ value) -1 , where 40 is the highest possible $C_{\mathrm{t}}$ attainable.

Calculation of sensitivity and specificity and statistical analysis. Sensitivity for PCR assay was calculated as the number of test (PCR assay) positives divided by the number of 'gold standard' (culture) positive strains multiplied by 100 . Specificity for PCR assay was calculated as the number of test (PCR assay) negatives divided by the number of 'gold standard' (culture) negatives multiplied by 100 .

PCR assay was capable of detecting both $C$. coli and C. jejuni at the same time, whereas by culture methods only one organism was detected based on one colony picked and identified. As such, it was not really possible to compare the sensitivity and specificity of PCR assay compared to culture for individual samples. The value for the sensitivity and sensitivity calculations therefore was determined on the basis of a positive or negative result for both or either species by either method.

To determine whether the different PCR assays gave comparable results, the $t$-test function of Microsoft Excel was used for statistical comparisons of $C_{\mathrm{t}}$ values for both FAM and HEX for different PCR assays of the same samples. FAM and HEX are the reporter dyes used on the probes for detection of C. jejuni and C. coli (Best et al., 2003). These dyes give rise to fluorescent signals for detection of either or both organisms.

PCR assays with no IAC, with the $16 \mathrm{~S}$ rDNA IAC and with the FV-IAC were compared for DNA extracts from 18 naturally infected caecal samples.

\section{RESULTS AND DISCUSSION}

\section{Initial optimization of IACs}

When the non-competitive $16 \mathrm{~S}$ rDNA IAC-PCR assay was used for pure culture lysates, there was no signal if the $16 \mathrm{~S}$ rDNA PCR primers were used at less than $1 / 2$ concentration, and the $16 \mathrm{~S}$ rDNA IAC signal strength was markedly reduced at $1 / 2$ concentration, so these primers were used at previously stated concentrations only. The $16 \mathrm{~S}$ rDNA IAC signals were generally lost in the presence of signals (unless $C_{\mathrm{t}}$ values were very high indicating weak signal) for either the C. coli or C. jejuni components of the PCR assay.

The FV-IAC caused inhibition of the PCR assay at dilutions of $10^{-5}$ and above (results not shown). At $10^{-6}$ dilution (20 pmol IAC), there was slight inhibition of weak signals (e.g. where $C_{\mathrm{t}}$ values were high; results not shown), but at $10^{-7}$ dilution (2 pmol IAC), there was no inhibition seen for either dilutions of cell lysates from cultures (Fig. 1) or for DNA extracts from 18 natural caecal samples (results not shown). A typical output for a sample positive for Campylobacter and for the competitive FV-IAC is shown in Fig. 2.

The effect of different dilutions of the Caecal PCR assay inhibitor solution on the signal from C. coli DNA is shown in Fig. 3. The Caecal PCR assay inhibitor solution was shown to be more potent at inhibiting the IAC signals for both the $16 \mathrm{~S}$ rDNA IAC and the FV-IAC than the signals from the C. coli or C. jejuni components of the PCR assay.

Our work was based on a duplex real-time PCR assay that had previously been carefully evaluated with a panel of cell lysates from 60 C. coli and C. jejuni strains, from negative control strains and from a further 5877 clinical isolates (Best et al., 2003). In view of the initial thorough nature of the PCR assay evaluation, our evaluation was on a smaller scale, but did show that both the competitive and noncompetitive IACs worked without reducing the sensitivity of the PCR assay. Additionally, both types of IACs were more sensitive to Caecal PCR assay inhibitor solution than the C. coli or C. jejuni components of the PCR assays, making them suitable for detecting inhibition and 


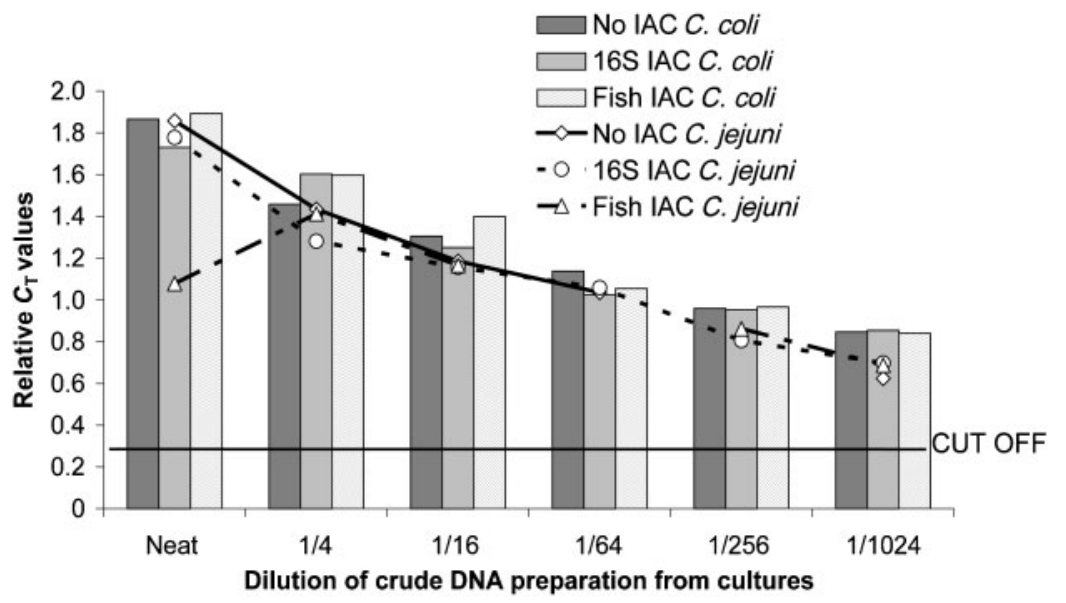

Fig. 1. Relative $C_{\mathrm{T}}$ values for dilutions of $C$. coli and C. jejuni cell lysates for duplex realtime PCR with and without IACs at optimum concentrations. preventing the recording of false-negatives (results not shown).

\section{Campylobacter numbers in spiked caecal samples}

The actual counts of Campylobacter in caecal samples spiked with both $C$. coli and C. jejuni from culture were as expected, i.e. giving $\sim \log _{10} 7,5,3,1$ c.f.u. $\mathrm{g}^{-1}$ for each Campylobacter species.

\section{Limit of detection}

The mean (SEM) DNA concentration for culture lysates from the seven positive and negative control cultures was 131 (17.3) ng $\mu l^{-1}$, whilst the mean (SEM) DNA concentration for 38 chicken caecal DNA extracts was 17 (4.6) $n g \mathrm{l}^{-1}$.

Culture lysates were still positive for C. coli and C. jejuni by PCR assay with and without the presence of both types of IACs when the DNA samples were diluted 1/1024 (Fig. 1). These results show that these PCR assays with and without IACs are capable of detecting Campylobacter DNA in

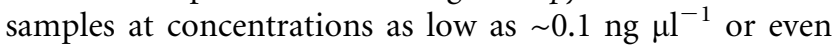

lower still, since at a dilution of $1 / 1024$ the $C_{\mathrm{t}}$ values for $C$. coli and $C$. jejuni were still in the range 21.6-24.6 (samples $>C_{\mathrm{t}} 32$ negative).

The spiked caecal samples were only positive when Campylobacter levels were at least $\log _{10} 5$ c.f.u. (g faeces) ${ }^{-1}$, and in most cases only positive with samples spiked with $\sim \log _{10} 7$ c.f.u. (g faeces) ${ }^{-1}$. This detection limit was not altered by the presence or absence of the different IACs.

The sensitivity of the PCR assay to detect Campylobacter in spiked chicken caecal samples was disappointing, especially when other workers report sensitivity down to $10^{3}$ c.f.u. (g faeces) ${ }^{-1}$ (Lin et al., 2008) or 36 c.f.u. $\mathrm{ml}^{-1}$ (Lund et al., 2003). However, in the method of Lund et al. (2003), the DNA extraction method included use of magnetic beads and this may have increased sensitivity over our method.

The primary aim of this work though was to develop an IAC sensitive to caecal inhibitors that would not affect the sensitivity of an existing PCR assay, rather than optimize the detection of Campylobacter from chicken faeces or caeca contents, and both IAC-PCR assays could be used with more efficient DNA extraction protocols. The titration of culture DNA extracts down to $1 / 1024$ showed that the addition of both of the IACs to the PCR assay did

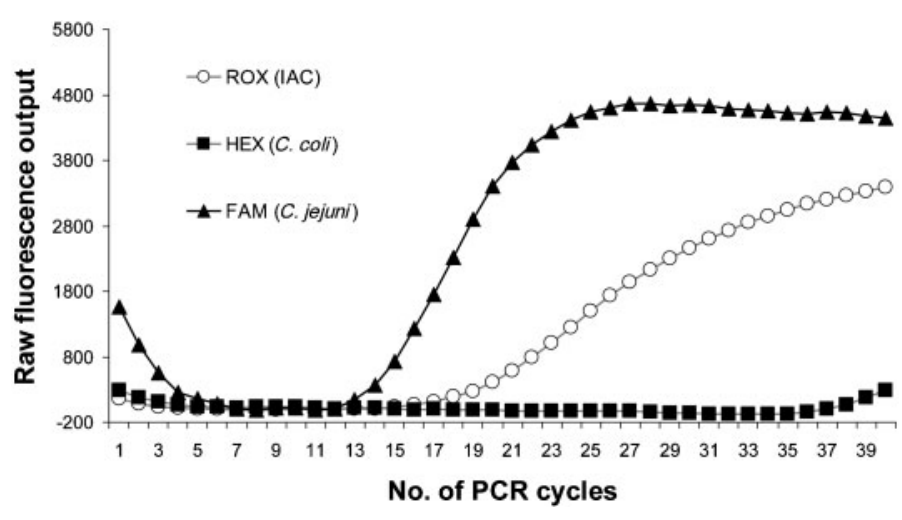

Fig. 2. Fluorescence readings for a sample positive for C. jejuni and for the FV-IAC. 


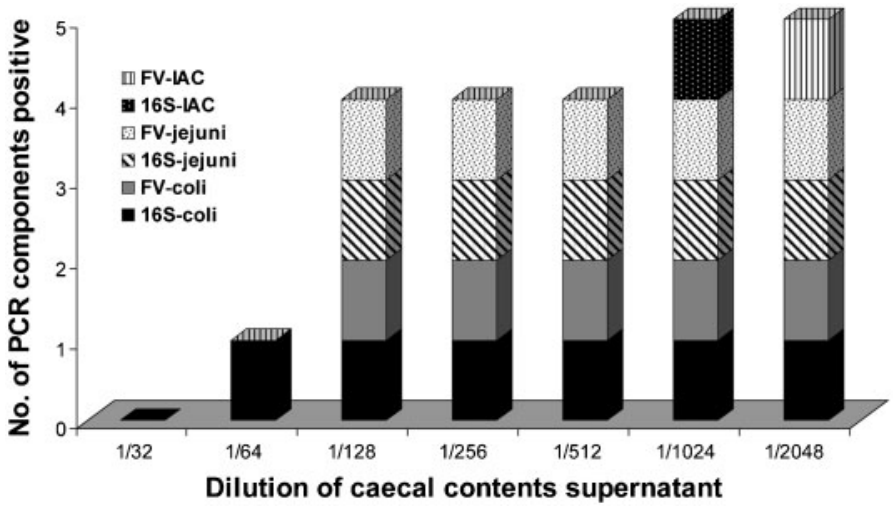

Fig. 3. Number of components of the 16SIAC and FV-IAC PCRs positive for increasing dilutions of caecal contents supernatant (PCR inhibitor). not reduce the sensitivity of the PCR assay and that the PCR assay was overall able to detect low levels of DNA. These results would suggest that the DNA extraction method rather than the PCR assay itself was the limiting factor in the overall sensitivity.

\section{Comparison of PCR with and without IACs for caecal contents}

When the PCR assays with no IAC, with the $16 \mathrm{~S}$ rDNA IAC and with the FV-IAC were compared for DNA extracts from 18 naturally infected caecal samples, there were no statistical differences between the $C_{\mathrm{t}}$ values for both FAM and HEX between any of the methods (results not shown).

With no IAC, 9/18, 14/18 and 7/18 samples were positive for C. coli, C. jejuni and both $C$. coli and C. jejuni, respectively. With the $16 \mathrm{~S}$ rDNA IAC, $9 / 18,15 / 18$ and $7 / 18$ samples were positive for $C$. coli, $C$. jejuni and both $C$. coli and $C$. jejuni, respectively. With the FV-IAC, 9/18, 16/18 and $9 / 18$ samples were positive for C. coli, C. jejuni and both C. coli and C. jejuni, respectively.

Results were similar for the PCR assay with and without the addition of the two different IACs and as such there was no evidence that the IACs were inhibiting detection of Campylobacter in chicken caecal samples as compared to the PCR assay without any IAC.

\section{Comparisons of FV-IAC PCR assay to culture}

Overall, the FV-IAC was considered the best IAC to use, so the FV-IAC PCR assay was used to compare with culture for 52 chicken caecal samples (Table 1).

The PCR identified 45/47 culture-positive samples as positive, with a $93.6 \%$ sensitivity and $80.1 \%$ specificity when comparing isolation or identification of both $C$. coli and C. jejuni (Table 1). However, an advantage of the PCR assay over the culture was that it was able to detect both $C$. coli and C. jejuni at the same time and within $24 \mathrm{~h}$, whilst only one colony from culture was tested to confirm one Campylobacter type and the minimum time for this was $48 \mathrm{~h}$. Thus PCR assay has the potential to be more rapid and, in a sense, more informative than culture. To obtain information on more than one Campylobacter species in caecal samples by culture would entail taking several colony picks from plates and this would become very timeconsuming.

The competitive FV-IAC was chosen to evaluate with a further 52 chicken caecal DNA extracts, since the signal for this IAC was not lost in the presence of strong signals from C. coli or C. jejuni, this FV-IAC did not rely on the presence of $16 \mathrm{~S}$ rDNA to work and since FV-IAC also did not have an extra set of primers which are reported to be able to cause problems by interacting with the target gene primers (Hoorfar et al., 2004). Additionally, previous authors state

Table 1. Comparison of culture to PCR with FV-IAC for natural chicken caecal samples $(n=52)$

\begin{tabular}{|lccc|}
\hline \multirow{2}{*}{ Sample test results } & \multicolumn{3}{c|}{ No. of samples } \\
\cline { 2 - 4 } & C. coli & C. jejuni & C. coli and C. jejuni \\
\hline Culture-positive & 10 & 38 & 0 \\
Culture-negative & 42 & 14 & 0 \\
PCR-positive & 22 & 39 & 15 \\
PCR-negative & 30 & 13 & 6 \\
& Organism & \% Sensitivity & \% Specificity \\
Culture/PCR & C. coli or C. jejuni & 93.6 & 80.1 \\
\hline
\end{tabular}

${ }^{\star}$ Culture was the 'gold standard' with which PCR was compared. 
that the non-competitive IACs have the disadvantage that amplification of non-competitive sequences may not accurately reflect amplification of the primary target due to differences in the primary sequences (Hoorfar et al., 2004).

Compared to culture, the PCR assay showed a good sensitivity and specificity for confirming the presence of Campylobacter in the 52 naturally infected caecal samples, and had the advantage of being able to identify the presence of both $C$. coli and C. jejuni simultaneously, which by culture would have been more laborious and would have required the testing of multiple colonies. A total of 15 samples were found to be positive for both $C$. coli and C. jejuni by PCR assay, only two samples confirmed positive by culture were negative by PCR assay and only one sample was PCR assay-positive but culturenegative.

Campylobacter concentrations in caecal contents of commercial chickens range from about 5 to $8 \log _{10}$ c.f.u. $\mathrm{g}^{-1}$ and when a flock is colonized birds can carry high numbers in their intestines (Mead et al., 1995; Rosenquist et al., 2006; Sahin et al., 2003; Stern et al., 1995; Stern \& Robach, 2003). Experimental studies where chickens were infected with 20 different strains of $C$. coli or $C$. jejuni showed most strains to colonize at levels of $\log _{10} 8-9$ c.f.u. $g^{-1} 2$ weeks after infection (Ziprin et al., 2003).

The 52 caecal samples that were tested for the presence of Campylobacter by culture compared to PCR assay were obtained at the time of year when numbers of Campylobacter shed from chickens are generally high, and this may have contributed to the good sensitivity of the PCR assay compared to culture, despite the apparent low detection rate using spiked samples. Different DNA extraction methods will be investigated to determine whether the detection threshold can be improved.

\section{Conclusions}

We have designed and evaluated both competitive and non-competitive IACs in a duplex real-time PCR assay for C. coli and C. jejuni and have shown both to work well for DNA from culture or from Campylobacter-infected chicken caecal content. The competitive FV-IAC is recommended since it does not rely on the presence of $16 \mathrm{~S}$ rDNA for a signal, it was not inhibited by C. coli or C. jejuni signals, it does not involve an extra set of primers that could interfere with the target gene primers and the signal derived from the FV-IAC should be detectable relative to the sample signal.

\section{REFERENCES}

Best, E. L., Powell, E. J., Swift, C., Grant, K. A. \& Frost, J. A. (2003). Applicability of a rapid duplex real-time PCR assay for speciation of Campylobacter jejuni and Campylobacter coli directly from culture plates. FEMS Microbiol Lett 229, 237-241.
Comi, G., Pipan, C., Botta, G., Cocolin, L., Cantoni, C. \& Manzano, M. (1996). A combined polymerase chain reaction and restriction endonuclease enzyme assay for discriminating between Campylobacter coli and Campylobacter jejuni. FEMS Immunol Med Microbiol 16, 45-49.

Deming, M. S., Tauxe, R. V., Blake, P. A., Dixon, S. E., Fowler, B. S., Jones, T. S., Lockamy, E. A., Patton, C. M. \& Sikes, R. O. (1987). Campylobacter enteritis at a university: transmission from eating chicken and from cats. Am J Epidemiol 126, 526-534.

Denis, M., Soumet, C., Rivoal, K., Ermel, G., Blivet, D., Salvat, G. \& Colin, P. (1999). Development of a m-PCR assay for simultaneous identification of Campylobacter jejuni and C. coli. Lett Appl Microbiol 29, 406-410.

Evans, S. J. (1992). Introduction and spread of thermophilic campylobacters in broiler flocks. Vet Rec 131, 574-576.

Genigeorgis, C., Hassuneh, M. \& Collins, P. (1986). Campylobacter jejuni infection on poultry farms and its effect on poultry meat contamination during slaughter. J Food Prot 49, 895-903.

Giesendorf, B. A., Quint, W. G., Henkens, M. H., Stegeman, H., Huf, F. A. \& Niesters, H. G. (1992). Rapid and sensitive detection of Campylobacter spp. in chicken products by using the polymerase chain reaction. Appl Environ Microbiol 58, 3804-3808.

Harris, N. V., Weiss, N. S. \& Nolan, C. M. (1986). The role of poultry and meats in the etiology of Campylobacter jejuni/coli enteritis. Am J Public Health 76, 407-411.

Hoorfar, J., Malorny, B., Abdulmawjood, A., Cook, N., Wagner, M. \& Fach, P. (2004). Practical considerations in design of internal amplification controls for diagnostic PCR assays. J Clin Microbiol 42, 1863-1868.

ISO (2006). Microbiology of Food and Animal Feeding Stuffs Horizontal Method for Detection and Enumeration of Campylobacter spp. Part 1: Detection Method. ISO 10272-1. Geneva: International Organization for Standardization.

Keramas, G., Bang, D. D., Lund, M., Madsen, M., Bunkenborg, H., Telleman, P. \& Christensen, C. B. (2004). Use of culture, PCR analysis, and DNA microarrays for detection of Campylobacter jejuni and Campylobacter coli from chicken feces. J Clin Microbiol 42, 3985-3991.

Kirk, R. \& Rowe, M. T. (1994). A PCR assay for the detection of Campylobacter jejuni and Campylobacter coli in water. Lett Appl Microbiol 19, 301-303.

Lawson, A. J., Logan, J. M., Neill, G. L., Desai, M. \& Stanley, J. (1999). Large-scale survey of Campylobacter species in human gastroenteritis by PCR and PCR-enzyme-linked immunosorbent assay. J Clin Microbiol 37, 3860-3864.

Lin, S., Wang, X., Zheng, H., Mao, Z., Sun, Y. \& Jiang, B. (2008). Direct detection of Campylobacter jejuni in human stool samples by realtime PCR. Can J Microbiol 54, 742-747.

Linton, D., Lawson, A. J., Owen, R. J. \& Stanley, J. (1997). PCR detection, identification to species level, and fingerprinting of Campylobacter jejuni and Campylobacter coli direct from diarrheic samples. J Clin Microbiol 35, 2568-2572.

Lund, M. \& Madsen, M. (2006). Strategies for the inclusion of an internal amplification control in conventional and real time PCR detection of Campylobacter spp. in chicken fecal samples. Mol Cell Probes 20, 92-99.

Lund, M., Wedderkopp, A., Waino, M., Nordentoft, S., Bang, D. D., Pedersen, K. \& Madsen, M. (2003). Evaluation of PCR for detection of Campylobacter in a national broiler surveillance programme in Denmark. J Appl Microbiol 94, 929-935.

Mead, G. C., Hudson, W. R. \& Hinton, M. H. (1995). Effect of changes in processing to improve hygiene control on contamination of poultry carcasses with Campylobacter. Epidemiol Infect 115, 495-500. 
Oyofo, B. A., Thornton, S. A., Burr, D. H., Trust, T. J., Pavlovskis, O. R. \& Guerry, P. (1992). Specific detection of Campylobacter jejuni and Campylobacter coli by using polymerase chain reaction. J Clin Microbiol 30, 2613-2619.

Rosenquist, H., Sommer, H. M., Nielsen, N. L. \& Christensen, B. B. (2006). The effect of slaughter operations on the contamination of chicken carcasses with thermotolerant Campylobacter. Int J Food Microbiol 108, 226-232.

Sahin, O., Kobalka, P. \& Zhang, Q. (2003). Detection and survival of Campylobacer in chicken eggs. J Appl Microbiol 95, 1070-1079.

Stern, N. J. \& Robach, M. C. (2003). Enumeration of Campylobacter $s p p$. in broiler feces and in corresponding processed carcasses. J Food Prot 66, 1557-1563.
Stern, N. J., Clavero, M. R., Bailey, J. S., Cox, N. A. \& Robach, M. C. (1995). Campylobacter spp. in broilers on the farm and after transport. Poult Sci 74, 937-941.

Wang, G., Clark, C. G., Taylor, T. M., Pucknell, C., Barton, C., Price, L., Woodward, D. L. \& Rodgers, F. G. (2002). Colony multiplex PCR assay for identification and differentiation of Campylobacter jejuni, C. coli, C. lari, C. upsaliensis, and C. fetus subsp. fetus. J Clin Microbiol 40, 4744-4747.

Wegmuller, B., Luthy, J. \& Candrian, U. (1993). Direct polymerase chain reaction detection of Campylobacter jejuni and Campylobacter coli in raw milk and dairy products. Appl Environ Microbiol 59, 2161-2165.

Ziprin, R. L., Sheffield, C. L., Hume, M. E., Drinnon, D. L. J. \& Harvey, R. B. (2003). Cecal colonization of chicks by bovine-derived strains of Campylobacter. Avian Dis 47, 1429-1433. 